\title{
Signet-ring Cell Carcinoma Component as an Indicator of Anaplastic Lymphoma Kinase Mutations in Colorectal Cancer
}

\author{
Suguru Nukada ${ }^{1224)}$, Yoichiro Okubo ${ }^{2)}$, Manabu Shiozawa ${ }^{1)}$, Emi Yoshioka ${ }^{2)}$, Masaki Suzuki ${ }^{2)}$ Kota Washimi ${ }^{2)}$, Kae Kawachi ${ }^{2)}$, \\ Sumito Sato ${ }^{1)}$ Yukihiko Hiroshima ${ }^{3)}$, Yasushi Rino ${ }^{4)}$, Tomoyuki Yokose ${ }^{2)}$ and Munetaka Masuda ${ }^{4)}$ \\ 1) Department of Gastrointestinal Surgery, Kanagawa Cancer Center, Yokohama, Japan \\ 2) Department of Pathology, Kanagawa Cancer Center, Yokohama, Japan \\ 3) Department of Cancer Genome Medicine, Kanagawa Cancer Center, Yokohama, Japan \\ 4) Department of Surgery, Yokohama City University, School of Medicine, Yokohama, Japan
}

\begin{abstract}
Objectives: Molecular profiling of marker mutations has become an essential aspect in the treatment planning for colorectal cancer (CRC). Anaplastic lymphoma kinase ( $A L K)$ mutations could be used as markers in CRC molecular profiling. However, the extremely low frequency of these mutations makes their confirmation in all patients inefficient. Thus, to determine whether ALK positivity could be indicated by morphological features, we have analyzed ALK positivity in CRC tissues with a signet-ring cell carcinoma (SRCC) component.

Methods: We screened cases of patients who underwent CRC surgical resection at the Department of Gastrointestinal Surgery of the Kanagawa Cancer Center between January 2015 and December 2019. The selected samples were then assessed immunohistochemically using an antibody against p80 ALK.

Results: In total, we were able to retrieve 29 cases of CRC with the SRCC component from the database; however, 5 cases were excluded owing to the absence of formalin-fixed paraffin-embedded tissue sections or the absence of the SRCC component when the tissues were observed. In the immunohistochemical analysis, two cases showed diffused positive immunoreactivity for ALK and were defined as ALK-positive CRC. Thus, the ALK positivity rate in CRC with SRCC was determined to be $8.3 \%$.

Conclusions: This present study sheds light on the morphological features of ALK-positive CRC. Our findings could contribute to the effective screening and improvement of front-line therapy for CRC.
\end{abstract}

\section{Keywords}

signet-ring cell carcinoma, anaplastic lymphoma kinase, colorectal cancer, immunohistochemistry, precision medicine

J Anus Rectum Colon 2021; 5(2): 167-172

\section{Introduction}

Colorectal cancer (CRC) has been identified as one of the major causes of morbidity and mortality worldwide. Although treatment outcomes have improved over the past 10 years owing to therapeutic advances[1], the prognosis of pa- tients with unresectable CRC remains unsatisfactory. In addition, the number of deaths owing to $\mathrm{CRC}$ has been on a steady increase; in fact, approximately 50,000 Japanese patients with CRC died in 2018[2,3].

The molecular profiling of $R A S, B R A F$, and microsatellites is considered an important modality in treatment plan- 
ning for CRC. However, to further improve treatment outcomes, individualized strategies based on more detailed genetic information are required. Previous studies have reported that anaplastic lymphoma kinase $(A L K)$ mutations are oncogenic drivers; their detection rate in patients with CRC ranges from $0.1 \%$ to $2.5 \%[4,5]$. These findings indicate that the timely confirmation of $A L K$ mutations in patients with CRC might lead to the early adoption of ALK tyrosine kinase inhibitors as treatment agents.

However, as the frequency of $A L K$ mutations is often extremely low, their confirmation in all patients can be inefficient. Therefore, we focused on the morphological features of $A L K$-rearranged lung adenocarcinoma. Of note, lung cancer is well known to be associated with $A L K$ mutations; particularly, an association between $A L K$ mutations and the signet-ring cell carcinoma (SRCC) component has been reported[6-8]. Furthermore, ALK tyrosine kinase inhibitors have been found to improve prognosis and therefore are of clinical importance in lung cancer harboring $A L K$ mutations[9]. Here, to detect $A L K$ mutations in patients with CRC, we screened cases of CRC using the SRCC component and confirmed ALK expression using immunohistochemistry (IHC).

\section{Methods}

\section{Selection of colorectal adenocarcinoma cases with an SRCC component}

In this retrospective study, data from patients treated at the Department of Gastrointestinal Surgery from the Kanagawa Cancer Center between January 2015 and December 2019 were collected and analyzed. We searched for cases using a pathological diagnosis support software ("WebPath" System, SEIKOTEC CO., LTD., Fukuoka, Japan). The terms "shoukakigeka-daicho" (Japanese for Department of Gastrointestinal Surgery), "signet ring," and "inkansaibou" (Japanese for signet ring) were used in retrieving CRC cases with the SRCC component. We subsequently extracted the following clinical data from the medical records of the retrieved cases for analysis: age, sex, primary site, treatment, outcome, and family or genetic predisposition.

Then, we reviewed the hematoxylin and eosin-stained slides of all the retrieved cases. Of note, in this study, all cases of CRC with an SRCC component were diagnosed as positive regardless of the proportion of the SRCC. We then calculated the proportion of the SRCC component in each case. This review was conducted by three oncologists (NS, YO, and TY).

\section{IHC and genetic analysis}

Tissue sections from tumors were embedded in paraffin and cut into $4-\mu \mathrm{m}$-thick sections. The sections were then de- paraffinized and pretreated with BOND Epitope Retrieval Solution 2 (EDTA, pH 9.0) for $30 \mathrm{~min}$. The Novocastra mouse monoclonal antibody p80 ALK (Leica Biosystems, clone 5A4) was diluted to $1: 100$ and was later incubated with the samples for $15 \mathrm{~min}$. Signals were detected using the BOND Polymer Refine Detection Kit (Leica Biosystems). ALK expression was assessed based on the percentage of cells with stained cytoplasm relative to that in controls and scored by three pathologists (NS, YO, and TY). IHC scores were assigned as 0 (no staining), 1 ( $0 \%<$ positive tumor cell rate $\leq 50 \%), 2(50 \%<$ positive tumor cell rate $\leq 80 \%)$, and $3(80 \%<$ positive tumor cell rate). An IHC score of 0 was regarded as negative, scores of 1 or 2 were considered borderline, and a score of 3 was considered positive. The positive cases were confirmed to contain the $A L K$ mutation by fluorescence in situ hybridization or comprehensive genomic profiling (FoundationOne CDx).

\section{Ethical approval}

The Ethics Committee of Kanagawa Cancer Center approved this retrospective study (Yokohama, Japan, approval no. 2020-EKI027).

\section{Informed consent}

Informed consent was obtained by opt-out in the study.

\section{Results}

\section{Clinicopathological data}

In total, 29 CRC cases with the SRCC component were retrieved from the database for analysis. The number of surgical specimens was 1362 (WebPath searched for "shoukakigeka-daicho" between January 2015 and December 2019), and the frequency of the SRCC component was $2.1 \%$. Five cases were excluded for the following reasons: the absence of formalin-fixed paraffin-embedded tissue sections and/or the absence of the SRCC component determined by microscopic observation.

Consequently, 24 cases were included in the study. The median age of the patients at the time of diagnosis was 67 (range, 27-78) years, and the male/female ratio was 2:1. Most primary tumors were determined to be located in the ascending colon $(n=10)$, followed by the appendix $(n=6)$, rectum $(n=4)$, transverse colon $(n=2)$, and descending and sigmoid colon $(\mathrm{n}=1)$.

The most common histologic type of main tissue was mucinous adenocarcinoma $(n=11)$, followed by poorly differentiated adenocarcinoma ( $\mathrm{n}=7)$, moderately differentiated adenocarcinoma $(\mathrm{n}=5)$, and SRCC $(\mathrm{n}=1)$. The average extent of the SRCC component was 12.1\% ( \pm 1 SD: 17.4), and the median was $7.5 \%$ (range: $1 \%-80 \%$ ). These findings are summarized in Table 1. 
Table 1. Clinical Characteristics of the Study Patients $(n=24)$.

\begin{tabular}{|c|c|c|}
\hline Age & Median (range) & $67(27-78)$ \\
\hline \multirow[t]{2}{*}{ Gender, n (\%) } & Male & $16(66.7 \%)$ \\
\hline & Female & $8(33.3 \%)$ \\
\hline \multirow[t]{7}{*}{ Primary site, $\mathrm{n}(\%)$} & Ascending colon & $10(41.7 \%)$ \\
\hline & Appendix & $6(25.0 \%)$ \\
\hline & Rectum & $4(16.7 \%)$ \\
\hline & Transverse colon & $2(8.3 \%)$ \\
\hline & Descending colon & $1(4.2 \%)$ \\
\hline & Sigmoid colon & $1(4.2 \%)$ \\
\hline & Cecum & $0(0 \%)$ \\
\hline \multirow[t]{4}{*}{ Stage, n $(\%)$} & I & $0(0 \%)$ \\
\hline & II & $0(0 \%)$ \\
\hline & III & $14(58.3 \%)$ \\
\hline & IV & $10(41.7 \%)$ \\
\hline \multirow[t]{4}{*}{ Histology, n (\%) } & Mucinous adenocarcinoma & $11(45.8 \%)$ \\
\hline & Poorly differentiated adenocarcinoma & $7(29.2 \%)$ \\
\hline & Moderately differentiated adenocarcinoma & $5(20.8 \%)$ \\
\hline & SRCC & $1(4.2 \%)$ \\
\hline \multirow[t]{2}{*}{ SRCC component } & Average & $12.1 \%( \pm 1 \mathrm{SD}: 17.4)$ \\
\hline & Median (range) & $7.5 \%(1 \%-80 \%)$ \\
\hline \multirow[t]{3}{*}{$R A S, \mathrm{n}(\%)$} & Wild type & $11(45.8 \%)$ \\
\hline & Mutations & $7(29.2 \%)$ \\
\hline & Unknown & $6(25.0 \%)$ \\
\hline \multirow[t]{3}{*}{$B R A F, \mathrm{n}(\%)$} & Wild type & $9(37.5 \%)$ \\
\hline & Mutations & $2(8.3 \%)$ \\
\hline & Unknown & $13(54.2 \%)$ \\
\hline
\end{tabular}

Abbreviations: MSI, microsatellite instability; SRCC, signet-ring cell carcinoma.

\section{ALK expression analysis via IHC}

Two cases of CRC with the SRCC component (Figure 1a, $\mathrm{b}$ and $\mathrm{d}$, e) showed diffusely positive immunoreactivity for ALK (both of them showed IHC scores of 3; $80 \%<$ positive tumor cell rate) and therefore were defined as $A L K$ positive CRC (Figure 1c and f). Furthermore, genetic analysis confirmed the EML4-ALK fusion in both cases. Both patients were males, with stage III tumors, which had a rapidly fatal course. Their relapse-free survival was 3 and 5 months, respectively, regardless of the administration of first-line chemotherapy. These findings are summarized in Table 2.

\section{Discussion}

Molecular-targeted therapies for CRC have advanced to play an essential role in improving the outcomes of patients with CRC. However, the current prognosis of patients with unresectable CRC has remained to be unsatisfactory. Therefore, further personalization of the therapeutic process, based on individual molecular profiling, is urgently required. Previous studies have demonstrated the potential therapeutic value of $A L K$ mutations in many solid tumors[10]. ALK in- hibitors have been applied for the treatment of patients with $A L K$-positive non-small cell lung cancer (NSCLC) in the clinical context, contributing to better outcomes[11]. Because the frequency of ALK mutations is extremely low, their confirmation in all patients might be inefficient. Recently, comprehensive genomic profiling (CGP) has been performed for refractory CRC patients, and ALK mutations could be detected more frequently. However, CGP has been found to be not suitable for early screening for ALK. We hypothesized that improving the detection rates of $A L K$ positive CRCs would, in turn, improve the prognosis of CRC via the early therapeutic introduction of ALK inhibitors. Therefore, we assessed whether a screening approach focusing on the SRCC component could improve the detection rates of $A L K$-positive CRCs.

A previous study showed that $A L K$-positive lung adenocarcinoma accounts for 3\%-5\% of NSCLC cases[12]. In this present study, 2 of the 24 cases $(8.3 \%)$ showed positive immunoreactivity for ALK; therefore, the detection rate of SRCC was substantially higher than the rate for all CRC reported in previous studies[4,5]. Of note, both $A L K$-positive cases in this study showed early multiple recurrence. Importantly, Bavi et al. reported that $A L K$ gene amplification is associated with poor prognosis in CRC[13], which is consis- 

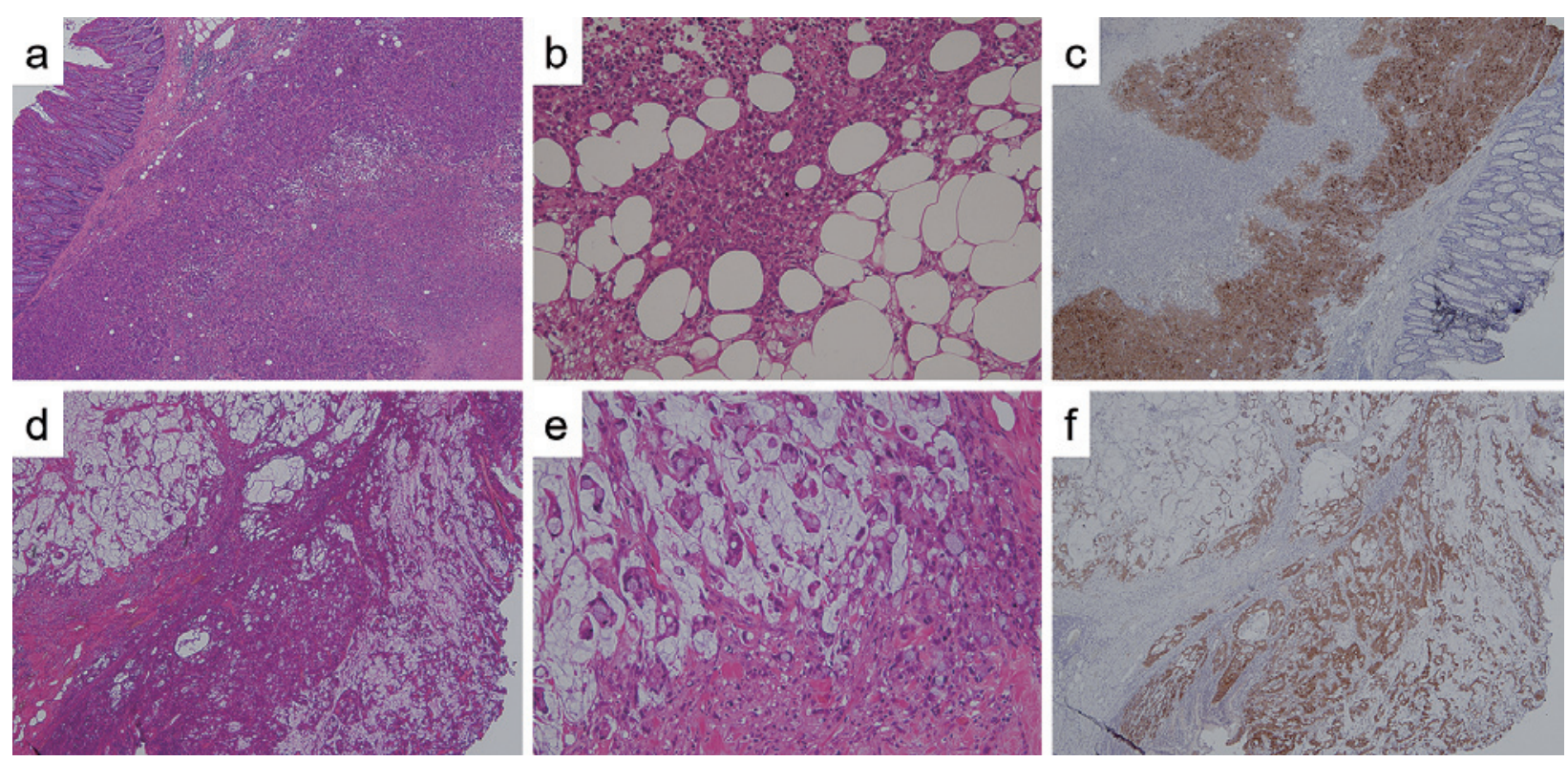

Figure 1. Histopathological findings of the two cases of colorectal adenocarcinoma with a signet-ring cell carcinoma (SRCC) component. (a) Carcinoma cells diffusely spread throughout the intestinal wall, with little or no glandular differentiation (Case 1, hematoxylin-eosin (HE) staining, $\times 40$ ). (b) A few SRCC components are observed in the outer zone of the tumor lesion adjacent to the subserosa (Case 1 , HE staining, $\times 200$ ). (c) Carcinoma cells show diffusely positive immunoreactivity for ALK (Case 1, immunohistochemistry, $\times 200$ ). (d) The intestinal wall is destroyed by carcinoma-producing mucin (Case 2, HE staining, ×40). (e) An abundant SRCC component floating within the mucin pool is observed (Case 2, HE staining, ×200). (f) Carcinoma cells show diffusely positive immunoreactivity for ALK (Case 2, immunohistochemistry, ×200).

Table 2. Clinicopathologic Characteristics of Colon Cancer Patients with Anaplastic Lymphoma Kinase Mutations.

\begin{tabular}{lcc}
\hline & Case 1 & Case 2 \\
\hline Age & 61 & 54 \\
Gender & Male & Male \\
Primary site & Rectum & Ascending colon \\
pTNM, stage & pT3N1bM0, Stage III B & pT4aN2aM0, Stage III C \\
Histology & Poorly differentiated adenocarcinoma & Mucinous adenocarcinoma \\
SRCC component & 2 & 10 \\
Adjuvant chemotherapy & TS-1 (2 courses) & CAPOX (6 courses) \\
Recurrent organ & Liver, lung & Liver, lung, peritoneum \\
Chemotherapy after recurrence & Bevacizumab plus FOLFOXIRI (14 courses) & Bevacizumab plus FOLFIRI (12 courses) \\
& Panitumumab plus FOLFOX (5 courses) & 5 \\
RFS (mo) & 3 & 16 \\
OS (mo) & 22 & Wild type \\
RAS & Wild type & Wild type \\
BRAF & Wild type & Stable \\
MSI & Stable & FISH: ALK positive \\
Genetic mutation & FoundationOne CDx: EML4-ALK, RNF43, TP53 & \\
\hline
\end{tabular}

Abbreviations: FISH, fluorescence in situ hybridization; RFS, relapse-free survival; OS, overall survival.

* Stage was defined according to the UICC 8th edition

tent with our findings. Among other genetic mutations, $R A S$ and $B R A F$ mutations did not show any specific frequency. Furthermore, although SRCC has been reported to be associated with MSI-high disease[14], two ALK-positive cases were MSS.
Further discussion is required on the morphological features of $A L K$-positive CRC. In NSCLC, a solid signet-ring cell pattern and a mucinous cribriform pattern are present, at least focally, in the majority (78\%) of $A L K$-positive tumors, but these are rare (1\%) in $A L K$-negative tumors[8]. Thus, we 
propose that an evaluation of the presence of the SRCC component via histology might help in identifying candidate cases for $A L K$ testing in CRC, considering that SRCC is a rare CRC subtype[14], accounting for 1\%-2.4\% of CRC cases[15]. By definition, signet-ring cell CRC is defined by the presence of at least 50\% of the SRCC component[14]. However, it has been reported that approximately 5\% of CRC cases have the SRCC component[16]. Furthermore, Rodig et al. reported that the majority of $A L K$-rearranged tumors $(56 \%)$ demonstrate a solid growth pattern, with an SRCC component of more than $10 \%$ in lung cancer[7]. In this present study, the median SRCC component was 7.8\%; of note, in the two cases with ALK protein expression, this was $2 \%$ and $10 \%$. However, owing to the small number of samples in this study, we could not observe any relevant association. Yet, even considering our small sample size, we could detect a relatively high rate of CRC harboring $A L K$ mutations with an SRCC component of less than $10 \%$. These results suggest that pathologists should report the presence of the SRCC component in CRC (regardless of its proportion) and that the SRCC component accounts for a substantial percentage of CRC.

In conclusion, this present study sheds light on the morphological features of $A L K$-positive CRC. These findings could contribute to effective screening and the improvement of front-line therapy for CRC. However, a few limitations of our study should be considered. The major one is that this retrospective study was based on a relatively small sample size. Therefore, further studies are required to support our observations.

Acknowledgements

We thank Ms. Sachie Osanai and Ms. Maho Sato for their excellent technical assistance.

\section{Conflicts of Interest}

There are no conflicts of interest.

\section{Source of Funding}

JSPS KAKENHI Grant Number JP17K08713 supported this work to Y. O. from the Ministry of Education, Culture, Sports, Science, and Technology of Japan.

\section{Author Contributions}

SN, YO, and TY contributed to the conception and design of the study. SN, YO, MSh, EY, MSu, KW, KK, and SS contributed to the data collection and assembly. SN and YO wrote the article. Initials of all authors contributed to the interpretation of the data and critical revision of the article. All authors have read and approved the final manuscript.

Approval by Institutional Review Board (IRB)

The Ethics Committee approved this retrospective study of Kanagawa Cancer Center (Yokohama, Japan, approval no. 2020-EKI027).

\section{References}

1. Yamazaki K, Nagase M, Tamagawa $\mathrm{H}$, et al. Randomized phase III study of bevacizumab plus FOLFIRI and bevacizumab plus mFOLFOX6 as first-line treatment for patients with metastatic colorectal cancer (WJOG4407G). Ann Oncol. 2016 Aug; 27(8): 153946.

2. Cancer Registry and Statistics. Cancer Information Service, National Cancer Center, Japan (Vital Statistics of Japan) [cited 2020 Jul 12]. Available from https://ganjoho.jp/reg_stat/statistics/dl/inde x.html\#mortality

3. Ishiguro M, Ueno H, Kanemitsu Y, et al. Current clinical practice of adjuvant chemotherapy for patients with 'high-risk' Stage II colorectal cancer in Japan: a questionnaire survey in the JCOG Study Group. Jpn J Clin Oncol. 2018 Dec; 48(12): 1109-12.

4. Aisner DL, Nguyen TT, Paskulin DD, et al. ROS1 and ALK fusions in colorectal cancer, with evidence of intratumoral heterogeneity for molecular drivers. Mol Cancer Res. 2014 Jan; 12(1): 111-8.

5. Yakirevich E, Resnick MB, Mangray S, et al. Oncogenic ALK fusion in rare and aggressive subtype of colorectal adenocarcinoma as a potential therapeutic target. Clin Cancer Res. 2016 Aug; 22 (15): 3831-40.

6. Jokoji R, Yamasaki T, Minami S, et al. Combination of morphological feature analysis and immunohistochemistry is useful for screening of EML4-ALK-positive lung adenocarcinoma. J Clin Pathol. 2010 Dec; 63(12): 1066-70.

7. Rodig SJ, Mino-Kenudson M, Dacic S, et al. Unique clinicopathologic features characterize ALK-rearranged lung adenocarcinoma in the western population. Clin Cancer Res. 2009 Aug; 15 (16): 5216-23.

8. Yoshida A, Tsuta K, Nakamura H, et al. Comprehensive histologic analysis of ALK-rearranged lung carcinomas. Am J Surg Pathol. 2011 Aug; 35(8): 1226-34.

9. Kwak EL, Bang YJ, Camidge DR, et al. Anaplastic lymphoma kinase inhibition in non-small-cell lung cancer. N Engl J Med. 2010 Oct; 363(18): 1693-703.

10. Siraj AK, Beg S, Jehan Z, et al. ALK alteration is a frequent event in aggressive breast cancers. Breast Cancer Res. 2015 Sep; 17: 127.

11. Li G, Dai WR, Shao FC. Effect of ALK-inhibitors in the treatment of non-small cell lung cancer: a systematic review and metaanalysis. Eur Rev Med Pharmacol Sci. 2017 Aug; 21(15): 3496503.

12. Mitsudomi T. Advances in target therapy for lung cancer. Jpn J Clin Oncol. 2010 Dec; 40(2): 101-6.

13. Bavi $\mathrm{P}$, Jehan $\mathrm{Z}, \mathrm{Bu} \mathrm{R}$, et al. ALK gene amplification is associated with poor prognosis in colorectal carcinoma. Br J Cancer. 2013 Nov; 109(10): 2735-43.

14. Korphaisarn K, Morris V, Davis JS, et al. Signet ring cell colorectal cancer: genomic insights into a rare subpopulation of colorectal adenocarcinoma. Br J Cancer. 2019 Sep; 121(6): 505-10.

15. Wang L, Hirano Y, Heng G, et al. Does signet ring cell carcinoma component signify worse outcomes for patients with colorectal cancer? Asian J Surg. 2020 Apr; 44(1): 105-10.

16. Pande R, Sunga A, Levea C, et al. Significance of signet-ring cells in patients with colorectal cancer. Dis Colon Rectum. 2008 Jan; 
51(1): 50-5. 